\title{
BMJ Open Protocol for a prospective longitudinal study of risk factors for hypertension incidence in a Mexico City population: the Tlalpan 2020 cohort
}

Eloisa Colín-Ramírez, ${ }^{1}$ Susana Rivera-Mancía, ${ }^{1}$ Oscar Infante-Vázquez, ${ }^{2}$
Raúl Cartas-Rosado, ${ }^{1}$ Jesús Vargas-Barrón, ${ }^{3}$ Magdalena Madero, ${ }^{4}$ Maite Vallejo ${ }^{5}$
To cite: Colín-Ramírez E, Rivera-Mancía S, InfanteVázquez 0 , et al. Protocol for a prospective longitudinal study of risk factors for hypertension incidence in a Mexico City population: the Tlalpan 2020 cohort. BMJ Open 2017;7:e016773. doi:10.1136/ bmjopen-2017-016773

- Prepublication history for this paper is available online. To view these files please visit the journal online (http://dx.doi org/10.1136/bmjopen-2017016773).

Received 9 March 2017 Revised 24 May 2017 Accepted 20 June 2017

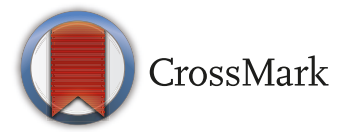

For numbered affiliations see end of article.

Correspondence to

Dr Maite Vallejo;

maite_vallejo@yahoo.com.mx

\section{ABSTRACT}

Introduction Systemic hypertension (HTN) is a common risk factor for cardiovascular disease. In Mexico, HTN prevalence has increased over time and is currently $31 \%$. Nonetheless, information about the country's HTN incidence and its associated risk factors is scarce. Understanding this condition is a priority for identifying the scope of primary prevention. The main objective of this study is to evaluate the effect of traditional and nontraditional risk factors on the incidence of HTN in a cohort of healthy Mexico City residents under biannual follow-up for 10 years.

Methods and analysis A prospective longitudinal study is proposed in which clinically healthy residents of Mexico City between 20 and 50 years old will be recruited; the participants will be evaluated every 2 years over a period of 10 years or until they develop HTN. Evaluations regarding sociodemographic, clinical, anthropometric, biochemical, diet, physical activity, stress, sleep quality, alcohol and tobacco consumption factors will be performed. The participants will be recruited from the 16 municipalities of Mexico City through promotional strategies aimed at the community and will be clinically evaluated at a tertiary care institution, Instituto Nacional de Cardiología Ignacio Chávez (National Institute of Cardiology Ignacio Chavez), located in Mexico City, Mexico. Sample size estimated for this study is 3436 , and the Cox proportional hazards model will be used to estimate HRs for the association between explanatory variables and HTN using both raw and adjusted data.

Ethics and dissemination This study was approved by the Institutional Bioethics Committee of the Instituto Nacional de Cardiología Ignacio Chávez (National Institute of Cardiology Ignacio Chavez) under number 13-802. Findings from this study will be disseminated through scientific papers and research conferences.

\section{INTRODUCTION}

Systemic hypertension (HTN) is a risk factor for cardiovascular and renal diseases (fatal and non-fatal), including myocardial infarction, stroke, atherosclerosis, aortic aneurysm, hypertensive heart diseases, peripheral artery disease, and cardiac and chronic renal insufficiency. ${ }^{1}$

\section{Strengths and limitations of this study}

- This is the first cohort project in Mexico aimed to study the impact of traditional and non-traditional risk factors for systemic hypertension (HTN), with emphasis on those less explored prospectively or those with no conclusive results in other cohorts.

- To our knowledge, this is the first study to evaluate the impact of an index comprising several social determinants of health (the Index of Social Development) on HTN incidence.

- This study will provide relevant information to feed into health promotion strategies for the prevention and control of HTN.

- Macrolevel factors such as air pollution, migration and health system will not be assessed in this study, and these may impact incidence of HTN.

- A probabilistic random sampling approach is not feasible for this cohort.

Data from 1980 and 2008 show that the worldwide prevalence of high blood pressure (systolic blood pressure $\geq 140 \mathrm{~mm} \mathrm{Hg}$ or diastolic blood pressure $\geq 90 \mathrm{~mm} \mathrm{Hg}$ ) has decreased in recent decades, from $33 \%$ to $29 \%$ in men and from $29 \%$ to $25 \%$ in women, as a result of government health interventions. This reduction is most evident in certain high-income countries such as the USA and in Europe. Conversely, the opposite effect is being observed in low-income countries. ${ }^{2}$ In countries such as Mexico, Paraguay and Venezuela, the prevalence of HTN is currently greater than $30 \% .^{3}$ Specifically, in Mexico the prevalence of HTN has increased gradually: from $26.6 \%$ in $1993,{ }^{4} 29.4 \%$ in $2000^{5}$ and $30.8 \%$ in $2006^{6}$ to currently $33.3 \%$ in men and $30.8 \%$ in women. ${ }^{7}$ Due to the discrepancy in HTN epidemiology across regions, it is pertinent to study the factors related to its development in countries where this public health problem continues to increase.

Although HTN is associated with several well-known factors such as age, sex, race, diet, 
obesity, sedentary lifestyle, excessive use of alcohol and stress, ${ }^{8}$ in Mexico the effect of such factors on the development of HTN has not been prospectively evaluated. Additionally, HTN development has been linked to other factors less understood, such as sleep disorders, ${ }^{9}$ social determinants, ${ }^{10}$ genetic variants ${ }^{11}$ and dyslipidaemia, ${ }^{12}$ all of which must be integrated to fully study and understand this disease, and to potentially identify new targets for the prevention and treatment of HTN.

The main objective of this study is to evaluate the effect of traditional and non-traditional risk factors on the incidence of HTN in a cohort of healthy Mexico City residents under biannual follow-up for 10 years.

\section{METHODS AND ANALYSIS Geographical context}

This is an observational, longitudinal, prospective study that will be conducted in Mexico City, Mexico. According to the 2010 Population and Housing Census, ${ }^{13}$ Mexico City (formerly Federal District) has a population of 8.851 million. The city is divided into 16 municipalities (geographical and political units) that have an unequal population distribution (eg, Iztacalco has 16444 inhabitants $/ \mathrm{m}^{2}$ and Magdalena Contreras 3070 inhabitants/ $\mathrm{m}^{2}$ ), with an enormous social, economic and commercial diversity that generates inequality and inequity within the colonies or neighbourhoods.

\section{Sample size calculation}

The formula for a time-to-event analysis was used for estimating the sample size of this study. ${ }^{14}$ For this purpose, we considered the relative risks (RR) reported by Pereira et $a l^{15}$ for the association between overweight $(\mathrm{RR}=2.05)$, education $(\mathrm{RR}=0.57)$ and ethanol consumption $(\mathrm{RR}=1.63)$ and incidence of HTN. This calculation rendered a total sample size of 256, 571 and 756, respectively. Additionally, we estimated a sample size based on the association between incidence of HTN and high sodium intake reported by Takase $e t a l^{16}(\mathrm{HR}=1.25)$ and sleep disorders $(\mathrm{HR}=1.58)$ reported by $\mathrm{Lin}$ et $a l^{9}{ }^{9}$ obtaining a sample size of 2643 and 629 participants, respectively. All these estimations were performed considering the lowest probability of event (HTN) by group of age reported by Ford and Cooper ${ }^{17}(26 \%)$ in a similar 10-year follow-up period.

Thus, taking into consideration the higher sample size estimated (2643) and assuming a two-tailed coefficient alpha of 0.05 , a power of $80 \%$ and a rate of loss to follow-up of $30 \%$, the total number of participants to recruit is 3436 .

\section{Study population}

The Tlalpan 2020 cohort will include women and men between 20 and 50 years old who live in Mexico City and who do not suffer from HTN. Individuals previously diagnosed with diabetes mellitus, dysthyroidism, cerebrovascular disease, ischaemic cardiomyopathy, acute coronary syndrome (ACS), cancer with an effect on survival, pregnant women and those taking medications that have an effect on blood pressure will be excluded, as will those with cognitive and mental disabilities.

\section{Recruitment of participants}

For participant recruitment, massive dissemination methods, such as social media (Facebook and Twitter), communication media (radio, television) and distribution of promotional material (brochures and posters) in health, community, work and cultural centres and schools located in the various delegations of Mexico City, will be used. Study visits and information processing will be performed by appointment at the Instituto Nacional de Cardiología Ignacio Chávez (INCICh) (National Institute of Cardiology Ignacio Chavez). During the initial visit (baseline), clinical, anthropometric, biochemical, dietetic, physical activity, stress and sleep quality evaluations will be conducted. In addition, information on sociodemographic data, personal and family pathological antecedents, and alcohol and tobacco consumption will be collected.

During the initial visit, patients who do not report having a diagnosis of diabetes or HTN but have an average systolic and/or diastolic blood pressure of $\geq 140 / 90 \mathrm{~mm}$ $\mathrm{Hg}^{18}$ or fasting glucose of $\geq 7 \mathrm{mmol} / \mathrm{L}^{19}$ will be eliminated from the study and referred to their clinic or health centre for management (figure 1).

\section{Follow-up and end of follow-up}

Each individual will be followed up for 10 years, with visits scheduled every 2 years in the INCICh. The evaluations and surveys conducted during the initial visit will be repeated at each of the follow-up visits. End of follow-up will occur after 10 years or when the participant develops HTN (primary outcome variable). The following are considered losses to follow-up: withdrawal from the study, death, ischaemic cardiomyopathy, ACS, cerebral vascular disease and permanent change of residence outside of Mexico City. To reduce the rate of loss to follow-up because of abandonment, participants will be contacted every 6 months via email to thank them for their participation in the study, keep them informed about its progress and update their contact information. Additionally, participants will be contacted by telephone every 12 months to verify whether they have been diagnosed with HTN and to again corroborate their contact information. Also, electronic seasonal postcards will be sent to all the study participants.

All participants will be asked to immediately notify the investigators should they be diagnosed with HTN at another facility at any time during the life of the study. These participants will be asked to schedule a visit at the INCICh prior to starting any medical treatment to confirm the diagnosis (according to criteria described in the Blood pressure assessment and HTN definition' section) and to complete the 'end of the study' visit. The date of disease onset will be considered as the date of confirmation of the diagnosis. For those participants presenting a hypertensive crisis between follow-up visits requiring immediate treatment, the HTN diagnosis will 


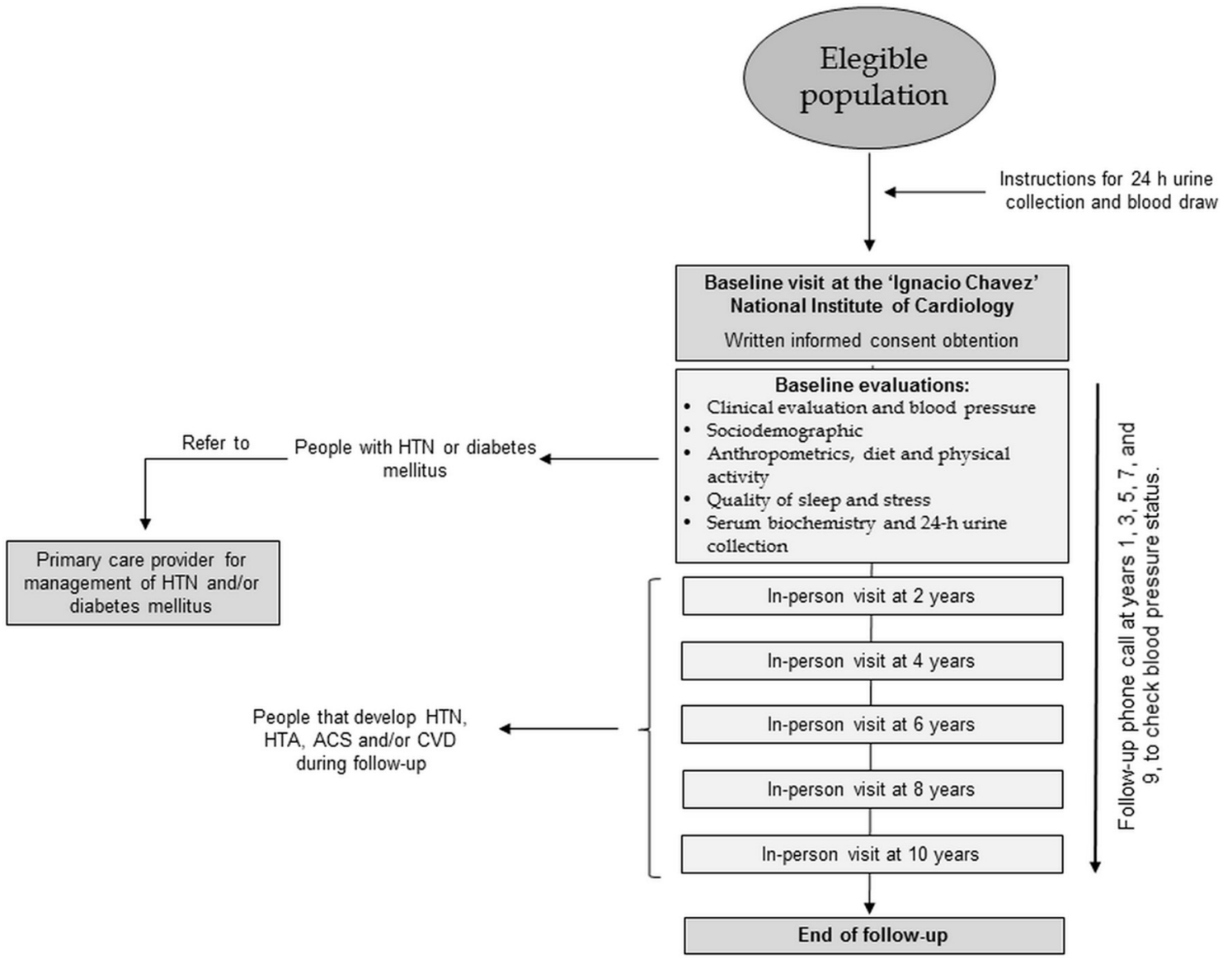

Figure 1 Tlalpan 2020 cohort scheme. All candidates to participate in the Tlalpan 2020 cohort receive the same instructions for their baseline visit; they are clinically evaluated to determine if they are included in the cohort and continue through the biannual follow-up visits. Those with hypertension or diabetes at the baseline visit are excluded from the cohort. For those included, the follow-up ends if they develop hypertension. ACS, acute coronary syndrome; CVD, cardiovascular disease; HTN, systemic hypertension.

be confirmed by medical records and then they will be scheduled for a clinical visit at the INCICh to complete the 'end of study' visit. The day of the hypertensive crises will be considered the date of disease onset. For those diagnosed with HTN during one of the biannual follow-up visits, the date of disease onset will be recorded as the date of the visit.

\section{Data capture and management}

Collection, capture and management of the information will be performed at the INCICh. Data are being captured through an electronic case report form (eCRF) specifically designed for this study. A dedicated Lenovo System x3500 M5 server will be used to store this information into a database structure, which will be managed using the Oracle Database 12c program. In order to establish remote connection between the server and the terminals used for collecting information on the eCRF, an HTMLbased application is being developed.

To minimise the risk of data loss, the server has a Redundant Array of Inexpensive Disks (RAID) 6.0 system and data backup on magnetic tape, as well as policies for incremental backups per day and full backups per week. Data backup ensures to recover either only the database in case it gets corrupted, or the full system in case of disaster.

\section{Evaluations and instrumentation}

\section{Blood pressure assessment and HTN definition}

Blood pressure will be measured in the left arm three times with a 3 min interval between each measurement, with the patient remaining in a sitting position for at least $10 \mathrm{~min}$ before the first evaluation. If one of the three measurements is quite different, another measure will be taken. The blood pressure value recorded will be the average of the three closer measurements. A cuff in accord with the diameter of the arm and a mercury sphygmomanometer, previously calibrated in the INCICh, will be used. HTN will be defined as a systolic blood pressure $\geq 140 \mathrm{~mm} \mathrm{Hg}$ and/or a diastolic blood pressure $\geq 90 \mathrm{~mm}$ Hg. ${ }^{18}$ Same diagnostic values will be applied for those cases being treated due to a hypertensive crisis and which HTN diagnosis will be confirmed by medical records. Heart rate (beats per minute) and respiratory rate (breaths per minute) will also be measured. 


\section{Anthropometry}

Evaluation of anthropometric measurements (weight, height and waist circumference) will be performed with the patient fasting, shoeless and wearing a hospital gown in accordance with the procedures described by 'The International Society for the Advancement of Kinanthropometry (ISAK)' ${ }^{20}$ For weight measurement, the patient will be asked to stand on the centre of the scale without support, with the arms hanging freely to the sides and with the weight distributed evenly on both feet. A mechanical column scale (SECA 700) with capacity of $220 \mathrm{~kg}$ and precision of $0.05 \mathrm{~kg}$ will be used and the weight will be recorded to the nearest $100 \mathrm{~g}$. Height will be measured with a stadiometer SECA 220, with the subject standing with the feet together, and the heels, buttocks and upper part of the back projected on the same vertical plane, with the head oriented on the plane of Frankfurt. Measurement will be taken at the end of a deep inward breath, placing the head board firmly down on the vertex, and recorded to the nearest millimetre. Waist circumferences will be measured at the level of the narrowest point (or at the midpoint if there is no obvious narrowing) between the lower costal border and the iliac crest by using a measuring tape made of glass fibre BodyFlex, with length of $150 \mathrm{~cm}$ and precision of $1 \mathrm{~mm}$. The patient will be instructed to breathe normally and the measurement will be taken at the end of a normal expiration.

\section{Sociodemographic indicators}

Using a structured questionnaire, information on age, sex, marital status, last grade of study (level of education), occupation and household monthly income will be obtained. Moreover, to evaluate the effect of the social component on the risk of HTN, the Índice de Desarrollo Social (IDS) (Index of Social Development), comprising quality and space of living, access to health and social security, educational gaps, durable goods, health, and energy adequacy, will be estimated. The IDS is constructed using information from the Population and Housing Census and updated with the most recent census and intercensal surveys. This index permits classifying the delegations, colonies and blocks of Mexico City according to their degree of social development in four strata; very low, low, medium and high. ${ }^{21}$

\section{Physical activity}

The long version ( 7 days) of the International Physical Activity Questionnaire (IPAQ), which was designed to assess physical activity in individuals 15-69 years old, will be used. The IPAQ measures the level of physical activity through questions regarding four domains: work, home, transportation and free time. The indicator of physical activity is expressed both continuously, in METs (metabolic equivalents)-minutes/week, and categorically, at levels of physical activity: low, moderate and high, according to the criteria of the IPAQ working group. $^{22}$

\section{Stress}

Stress will be evaluated using the State-Trait Anxiety Inventory (STAI), ${ }^{23}$ Spanish version. This instrument is designed to evaluate two forms of anxiety: state anxiety (A-State, referring to a transitory emotional condition) and trait anxiety (A-Trait, referring to a relatively stable anxious propensity). Participants respond to each of the STAI items on a scale of four categories. The categories for the A-State scale are (1) not at all, (2) somewhat, (3) moderately so and (4) very much so. The categories for the A-Trait scale are (1) almost never, (2) sometimes, (3) often and (4) almost always. For both scales, the minimum score is 20 and the maximum is 80 . A higher score indicates a higher level of anxiety.

\section{Sleep disorders}

To evaluate sleep disorders, the Spanish-language Medical Outcomes Study-Sleep scale of 12 items will be used to assess the last week of sleep. This scale was originally developed in the 'Medical Outcomes Study (MOS)' to evaluate relevant components of sleep in seven dimensions: sleep disturbances, sleep adequacy, sleep quantity, optimal sleep (dichotomous scale), somnolence, snoring, and sleep short of breath or headache. The scale also summarises sleep problems in two indices: index-1 consists of six items and index-2 nine items. During the evaluation, the participant will be asked to answer the questions with regard to the last week. In the case of the following domains - sleep disturbances, somnolence, snoring, shortness of breath/headache, and the two indices of sleep problems - their corresponding scores will be transformed into a 0-100 scale, where higher scores will reflect more of the specific problem. The sleep quantity dimension is the average number of hours a patient reports to sleep per night, while the optimal sleep dimension is a dichotomous scale that classifies the hours of sleep as optimal (between 7 and 8 hours of sleep) or not (less than 7 or more than 8 hours of sleep). ${ }^{24} 25$

\section{Dietary intake}

Dietary intake will be evaluated using a semiquantitative questionnaire for the frequency of consumption of food and beverages designed and validated in a Mexican population. This instrument includes 116 questions and evaluates the frequency of consumption of a standard portion of each food or beverage during the past year. The estimations of energy, fibre, macronutrient (proteins, carbohydrates and lipids) and micronutrient (vitamins and minerals) intakes will be performed using the computer program 'Sistema de Evaluación de Hábitos Nutricionales y Consumo de Nutrimentos' (SNUT) (System of Evaluation of Nutritional Habits and Nutrient Consumption), which was developed by the National Institute of Public Health of Mexico. ${ }^{26} 27$ Sodium and potassium intake will also be estimated by 24-hour urinary sodium and potassium excretion.

\section{Serum lipid profile}

Fasting total cholesterol (TC), high-density lipoprotein (HDL) and low-density lipoprotein (LDL) cholesterol, 
and triglycerides (TG) will be determined. Altered lipid profile will be defined according to the Adult Treatment Panel III criteria as follows: high TC when $>5.2 \mathrm{mmol} / \mathrm{L}$, low HDL-C when $<1.0 \mathrm{mmol} / \mathrm{L}$ for men and $<1.3 \mathrm{mmol} / \mathrm{L}$ for women, high LDL-C when $>3.4 \mathrm{mmol} / \mathrm{L}$ and high TG when $1.7 \mathrm{mmol} / \mathrm{L}{ }^{28}$

All instruments will be applied by personnel trained for this purpose. During the structured surveys, information about personal and family pathological antecedents and alcohol (frequency and amount habitually consumed) and tobacco use will be collected. Participants who at the time of the survey report to consume alcohol regardless of frequency of intake (daily, every other day, every weekend, every 2 weeks, once a month or less than once a month) will be considered as current alcohol consumers. Participants who report to smoke at least 100 cigarettes in their lifetime and who, at the time of survey, smoked either every day or some days will be classified as current smokers. ${ }^{29}$

\section{Collection, management and storage of biological samples 24-hour urine}

Detailed information will be provided to the participants regarding the correct way to carry out the collection (discard the first urine in the morning and collect all urine for a period of 24 hours, including the first urine of the following morning, which will be the day of the visit). Participants will be provided with a preservative-free container and asked to keep the collected urine in a cool place during the collection period. Urinary sodium and potassium will be determined by the ion selective electrode method, and urinary creatinine will be determined by Jaffe's colorimetric assay using an automated analyser. The urine sample will be considered to be complete when urinary creatinine levels are within the standard creatinine excretion rate for each sex (133-221 $\mu \mathrm{mol} /$ $\mathrm{kg} / 24$ hours for men and $88-177 \mu \mathrm{mol} / \mathrm{kg} / 24$ hours for women). ${ }^{30}$

\section{Blood samples}

A $30 \mathrm{~mL}$ sample of venous blood will be obtained from each participant after 12-hour fasting period. To separate the plasma, the sample will be placed in tubes with EDTA; to separate the serum, tubes without anticoagulant will be used. Haematic biometry will be performed on whole blood; serum will be used for determination of the levels of glucose, uric acid, creatinine, sodium and lipid profile.

The cellular packet from those participants who grant their consent for genetic substudy will be used for extraction of genomic DNA using the technique of Lahiri and Nurnberger ${ }^{31}$ and subsequent determination of polymorphisms associated with HTN development.

\section{Storage of biological samples}

Aliquots of serum, plasma, urine and DNA will be prepared as described in figure 2. All aliquots will be stored at $-70^{\circ} \mathrm{C}$ for a period of 25 years after the end of follow-up of each participant. Each aliquot will only be labelled with the identification number of the subject, and its location will be electronically recorded in the
eCRF using a coordinate system that includes the position in the cryobox in the rack, the level in the ultrafreezer and the number of the ultrafreezer (figure 2). Aliquots of all sample types will be divided into two equal subsets, each one stored in a different ultrafreezer for backup of the samples at all times.

\section{Genetic substudy}

On completion of study follow-up, aliquots of DNA samples corresponding to incident cases of HTN and to the participants who have not developed the disease will be analysed, matching by sex and age. Haplotypes will be examined based on single-nucleotide polymorphisms using the TaqMan $5^{\prime}$ exonuclease genotyping assay. ${ }^{32}$ However, because significant progress may be achieved in the field of genetic analysis in the coming years, the technologies existing at the time of analysis will be taken into consideration. Currently, the study of gene haplotypes of the renin-angiotensin-aldosterone system and the autonomic nervous system is considered. Nonetheless, findings reported in the literature at the time of follow-up completion will be taken into account, including those related to other genes that may be of interest for explaining the development of HTN.

\section{Quality control and validation of the information}

The operating staff will receive training in performing clinical procedures (measuring blood pressure and anthropometric parameters), surveys and data collection to ensure their competence and standardisation. Periodically, blood pressure measurements will be obtained by two independent observers in a subsample of participants to verify the reproducibility of the measurement procedure.

Data entry will be performed continuously for updating the database and running quality control analysis. Outliers or aberrant data in all of the captured data will be identified bimonthly, and at least $5 \%$ of the data will be randomly selected and checked against the source document for validation.

All measurement equipment (scales and sphygmomanometers) will be calibrated every 6 months by a team of biomedical engineers at the INCICh in accordance with the European Society of Hypertension International Protocol. ${ }^{33}$

\section{Statistical analysis of the data}

For the purposes of data analysis, the primary response variable (outcome) will be HTN, and the explanatory variables will be sociodemographic, hereditary antecedent, anthropometric, biochemical, genetic, dietetic, physical activity, smoking, stress and sleep quality factors. Those variables that have been shown to be strongly associated with blood pressure, such as sex, age and alcohol consumption, will initially be considered confounding variables. ${ }^{3435}$

Descriptive analysis of the baseline characteristics of the overall population and by gender will be conducted. Categorical variables will be presented as relative and absolute frequencies and continuous variables as the mean $\pm \mathrm{SD}$ 


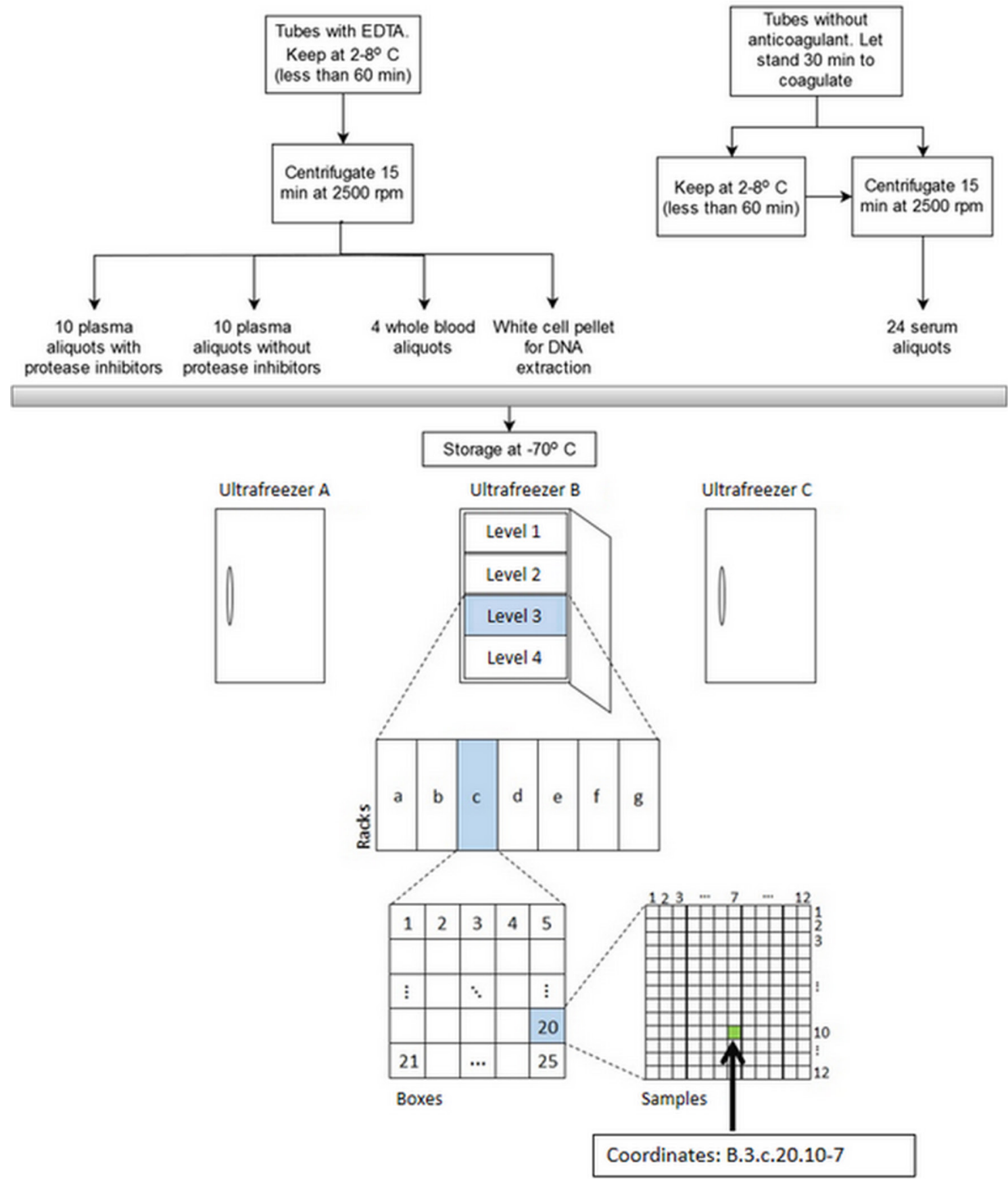

Figure 2 Processing and storage procedure for biological samples of participants. After blood draw, plasma and serum are obtained, aliquoted and stored at $-70^{\circ} \mathrm{C}$ in ultrafreezers. A coordinates system based on a combination of letters and numbers that identify each ultrafreezer, its level and rack, as well as the row and column in the cryobox, was developed to store all samples.

or median (25-75 percentile), depending on the nature of their distribution. For the comparison of categorical variables between the sexes, Pearson's $\chi^{2}$ test will be used (or Fisher's exact test when at least $20 \%$ of the cells have expected frequencies less than five). In the case of continuous variables, parametric and non-parametric methods will be employed, depending on the distribution of the data. The incidence rate of HTN will be estimated as the number of new cases of HTN over the total person-time at risk. The person-time at risk for each individual will be estimated as the time each participant remains free of HTN during the follow-up period (10 years). The HTN incidence rate will be calculated for the overall population and stratified by sex.

The primary analysis will be based on the estimation of HR and 95\% CIs to quantify the magnitude of the association between explanatory variables and the incidence of HTN. This analysis will be conducted using the Cox proportional hazards method, and the HR and 95\% CI will be estimated for the association between explanatory 
variables and the development of HTN both crude and adjusted by age, sex, alcohol consumption and other variables identified as potential confounders. Additionally, multiple linear regression analysis will be used to evaluate the association between biannual changes in continuous explanatory variables and biannual changes in blood pressure, adjusting for confounding variables.

The existence of non-linear effects of continuous variables on blood pressure will also be evaluated, and possible interactions between variables defined a priori (sex, age and alcohol consumption, among others that are of interest) will be examined. Non-linear effects may occur as quadratic or higher order variables or as interactions between variables. If any of these non-linear terms are significant, the effects will be included in the final models.

Missing data from explanatory variables will be handled by the multiple imputation method. Participants lost to follow-up will be treated as right-censored data, considering the date of the last contact.

To assess the potential bias associated with loss to follow-up, a sensitivity analysis will be performed in which baseline characteristics between the participants who complete follow-up and those who do not will be compared. Characteristics that are identified as different between these groups will be included as covariables in the models. A $p$ value of $<0.05$ will be considered statistically significant for all analyses.

For the genetic substudy, Hardy-Weinberg equilibrium analysis will be performed. Conditional logistic regression analysis will then be conducted to detect associations between polymorphisms or haplotypes with HTN. Four inheritance models will be considered for this analysis: dominant, codominant, recessive and heterozygote advantage. The Akaike information criterion will be used to select the most appropriate model. Subsequently, prediction models for HTN will be constructed using conditional logistic regression analysis based on the inheritance model selected, including anthropometric, lifestyle and biochemical indicators as explanatory variables.

\section{Pilot study}

To test the instruments and procedures for the collection of information, a pilot study was conducted on a sample of 70 individuals enrolled in a health centre located in the Tlalpan municipality. For this pilot test, the same selection criteria were employed, except that instead of residents of any municipality of Mexico City, only residents of the Tlalpan municipality were included. During this pilot study, problems in the language used in the interviews, in the scope of the recruitment strategies and in the sequence of procedures during the visit were clearly identified. Therefore, after this pilot phase, terms identified as unclear to participants used during the face-to-face interview were revised, and the use of mass media and social media was included in the recruitment strategies. Moreover, the logistics regarding the processes of measurement and data collection were reorganised into four main steps: (1) reception of participants, (2) collection of biological samples, (3) clinical and anthropometric measurements, and (4) evaluations of life habits and face-to-face interviews. The total time required to complete a clinical visit was estimated at approximately 2 hours per participant, and a capacity of receiving up to 20 participants per day was determined.

\section{DISCUSSION}

HTN is associated with several well-known factors such as age, sex, race, diet, obesity, sedentary lifestyle, excessive use of alcohol and stress, ${ }^{8}$ and other less understood such as sleep disorders, social determinants, genetics and dyslipidaemia. Tlalpan 2020 is the first study to establish the role of traditional and non-traditional risk factors in the development of HTN in a Mexican population.

The association between sleep duration and HTN has been suggested in longitudinal ${ }^{9} 36-41$ and cross-sectional studies, ${ }^{42-45}$ without consistent conclusions. Importantly, most of these studies have focused on duration of sleep without considering other sleep disorders. The present study will include the assessment of diverse aspects of sleep beyond its quantity. Regarding social determinants, they refer to the conditions in which people are born, grow, work, live and age, and the set of conditions of their daily life. ${ }^{46}$ It has not been until the last years that the impact of social determinants on health has attracted the attention of a broader community research and government institutions. ${ }^{46}$ In the case of HTN, its association with social determinants has been more explored in the context of prevalence ${ }^{47-50}$ than incidence. ${ }^{51-53}$

In the genetics field, although recent discoveries have shown more convincing evidence regarding the relationship between genetic variants and $\mathrm{HTN},{ }^{11}$ more studies exploring the interactions between genes and the environment are still needed. The study of the influence of the genetic variants discovered in the last years and those arisen in the future on the development of HTN, and their interaction with other factors being evaluated in this study, will provide useful information for the prevention and management of this disease in our population.

With respect to metabolic factors, the coexistence of dyslipidaemia and HTN is common ${ }^{54-56}$; however, the effect of dyslipidaemia on incidence of HTN has been little explored, ${ }^{12}$ and studies in this topic have been performed only in men $^{57}$ or in the elderly. ${ }^{58}$ Importantly, abnormalities in lipid profile in Mexican population are highly prevalent: data from the National Health and Nutrition Survey 2006 showed low HDL-cholesterol in $60.5 \%$ of the sample, high TC in $43.6 \%$ and hypertriglyceridaemia in $31.5 \% .^{59}$

Additionally, the relationship between excessive sodium intake and high blood pressure has been widely recognised ${ }^{60}$; however, information on sodium consumption in the Mexican population is limited. The data derived from this study will permit us to estimate sodium 
intake and assess its contribution to the development of HTN in this population.

According to the information discussed above, the need of studying all of these aspects in an integral and prospective manner to better understand their impact on HTN development is evident.

Although a probabilistic random sampling approach is not feasible in the Tlalpan 2020 cohort, this study is the first cohort in Mexico aimed to prospectively examine the impact of traditional and non-traditional risk factors on HTN development. Importantly, an index comprising several social determinant of health (IDS) will be used for the first time to evaluate their role on HTN incidence. This study will also permit the creation of a biobank accessible to the scientific community studying genetic and biochemical factors involved in the development of cardiovascular diseases. Tlalpan 2020 is expected to be a precedent for a future study that meets national efforts to estimate the incidence of HTN in Mexico and will provide relevant information to feed into health promotion strategies for the prevention and control of HTN.

The first participant was enrolled on 11 September 2014. As shown in figures 3, 1524 candidates were evaluated for eligibility through August 2016; of these, 1397 $(91.6 \%)$ met the selection criteria and $127(8.4 \%)$ were eliminated after the initial visit because they did not meet the eligibility criteria. By that time, nearly 94000 aliquots of serum, plasma, urine, whole blood and DNA had been stored. The recruitment of participants is estimated to conclude by July 2018 .

\section{ETHICS AND DISSEMINATION \\ Ethical aspects}

Within the ethical framework, this research will be governed by the seventh revision of the Declaration of Helsinki $^{61}$ and the Regulations of the General Health Law in Matters of Research for Health of Mexico. ${ }^{62}$

This study was approved by the Institutional Bioethics Committee of the INCICh under number 13-802. Written informed consent will be obtained from all participants for inclusion in the main study and the genetic substudy.

Because the study proposes to obtain genetic material and its analysis could establish a possible association of genetics with the outcome variable, whether we inform the participant of the existence of this condition or genetic predisposition will follow the recommendations of the working group of the National Heart, Lung, and Blood Institute of the USA. ${ }^{63}$

\section{Data protection}

To protect the identity of the participants, each of them will be assigned a unique identification number in the study, which will be linked to their personal data. Access to the information will be managed using security controls for groups with different levels of privileges. These controls ensure the confidentiality of sensitive data, in accordance with the Official Mexican Standard

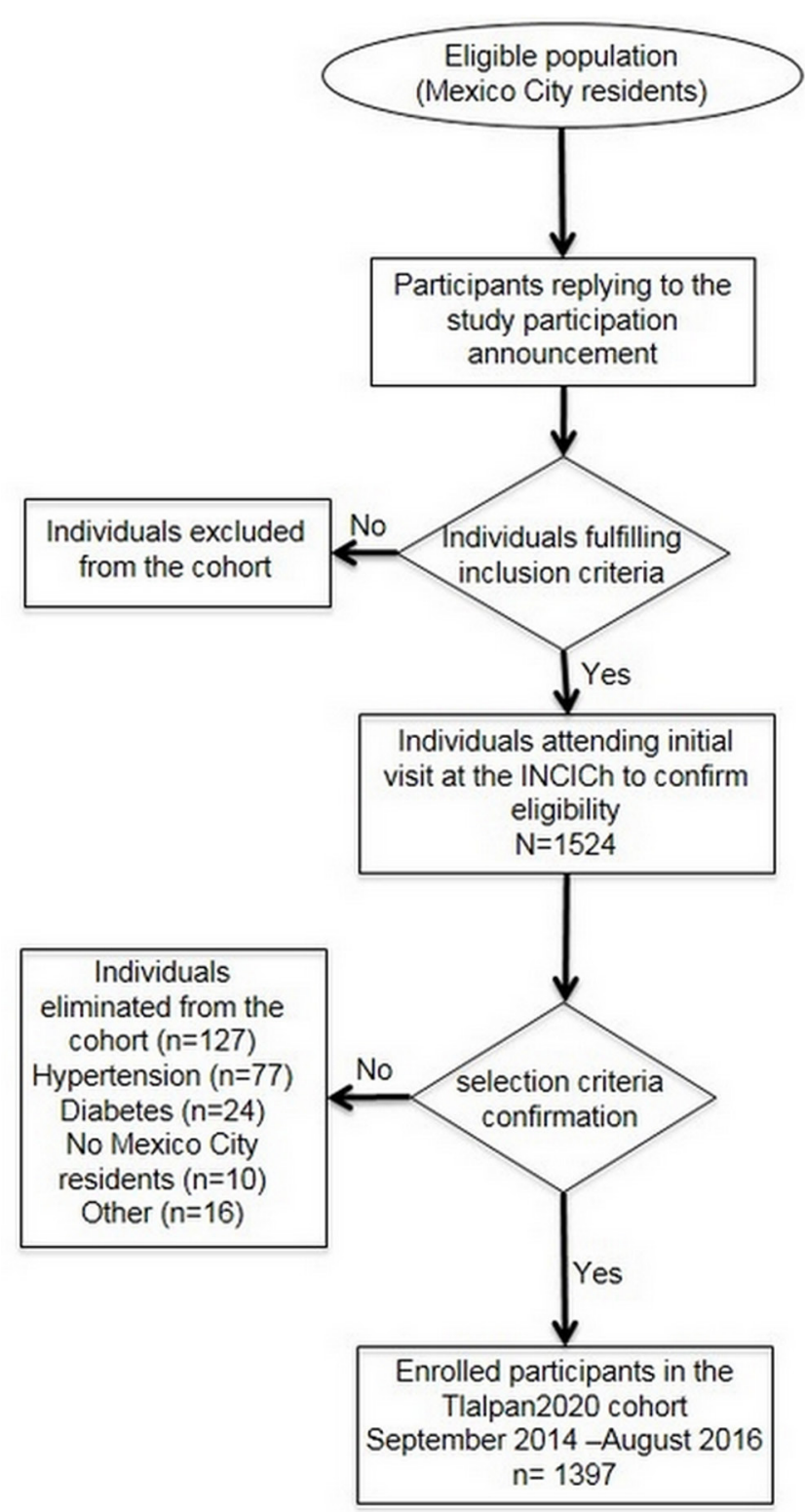

Figure 3 Participants flow chart in the Tlalpan 2020 cohort from September 2014 to August 2016. Since the beginning of the recruitment, 1524 persons have been evaluated for eligibility, 1397 of whom met the inclusion criteria for the Tlalpan 2020 cohort. INCICh, Instituto Nacional de Cardiología Ignacio Chávez.

NOM-024-SSA3-2012, 'Electronic record Information systems for health. Information exchange in health' and the Regulations of the General Health Law in Matters of Research for Health of Mexico. ${ }^{62}$

\section{Dissemination}

Results from this study will be disseminated through research papers and national and international conferences.

\section{Author affiliations}

${ }^{1}$ National Council for Science and Technology (CONACYT) - National Institute of Cardiology 'Ignacio Chávez', Juan Badiano 1, Sección XVI, Mexico City, Mexico 
${ }^{2}$ Department of Electromechanical Instrumentation, National Institute of Cardiology 'Ignacio Chávez' Juan Badiano 1, Sección XVI, Mexico City, Mexico

${ }^{3}$ Division of Research, National Institute of Cardiology 'Ignacio Chávez' Juan Badiano 1, Sección XVI, Mexico City, Mexico

${ }^{4}$ Department of Nephrology, National Institute of Cardiology 'Ignacio Chávez' Juan Badiano 1, Sección XVI, Mexico City, Mexico

${ }^{5}$ Department of Social Medicine Research, National Institute of Cardiology 'Ignacio Chávez', Juan Badiano 1, Sección XVI, Mexico City, Mexico

Acknowledgements The authors wish to thank the participants of this study. Contributors ECR contributed to the study design and drafted the manuscript; SRM contributed to design the system of management and storage of biological samples and drafted the manuscript; OIV contributed to the design and conception of the study; RCR contributed to the design of the data capture system and drafted the corresponding section of the manuscript; JVB contributed to the design and conception of the study; MM contributed to the design and conception of the study; MV contributed to the conception and design of the study and drafted the manuscript. In addition, all authors read and approved the final manuscript.

Funding This project is funded by the Consejo Nacional de Ciencia y Tecnología (CONACYT) (National Council for Science and Technology) under the 'Cátedras CONACYT' programme, No 1591. Funding was also received from AstraZeneca Mexico (collaboration agreement without number).

\section{Competing interests None declared.}

Patient consent This manuscript does not contain any personal medical information about an identifiable living individual. Data from participants of this study are anonymised. Each participant signs the informed consent approved by the Institutional Bioethics Committee of the Instituto Nacional de Cardiología Ignacio Chávez (National Institute of Cardiology Ignacio Chavez) under number 13-802.

Ethics approval This study was approved by the Institutional Bioethics Committee of the INCICh under number 13-802. Written informed consent will be obtained from all participants for inclusion in the main study and the genetic substudy.

Provenance and peer review Not commissioned; externally peer reviewed.

Open Access This is an Open Access article distributed in accordance with the Creative Commons Attribution Non Commercial (CC BY-NC 4.0) license, which permits others to distribute, remix, adapt, build upon this work non-commercially, and license their derivative works on different terms, provided the original work is properly cited and the use is non-commercial. See: http://creativecommons.org/ licenses/by-nc/4.0/

(C) Article author(s) (or their employer(s) unless otherwise stated in the text of the article) 2017. All rights reserved. No commercial use is permitted unless otherwise expressly granted.

\section{REFERENCES}

1. Rahimi K, Emdin CA, MacMahon S. The epidemiology of blood pressure and its worldwide management. Circ Res 2015;116:925-36.

2. Danaei G, Finucane MM, Lu Y, et al. National, regional, and global trends in fasting plasma glucose and diabetes prevalence since 1980: systematic analysis of health examination surveys and epidemiological studies with 370 country-years and $2 \cdot 7$ million participants. Lancet 2011;378:31-40.

3. Kearney PM, Whelton M, Reynolds K, et al. Global burden of hypertension: analysis of worldwide data. Lancet 2005;365:217-23.

4. Secretaría de Salud. Encuesta Nacional de enfermedades crónicas. 1993. https://www.insp.mx/encuestoteca.html.

5. Olaiz G, Rojas R, Barquera S, et al. Encuesta Nacional de Salud 2000. 2: la Salud de los adultos. Cuernavaca, Morelos: Instituto Nacional de Salud Pública, 2003.

6. Olaiz-Fernández G, Rivera-Dommarco J, Shamah-Levy T, et al. Encuesta Nacional de salud y nutrición. Cuernavaca, Morelos: Instituto Nacional de Salud Pública, 2006.

7. Campos-Nonato I, Hernández-Barrera L, Rojas-Martínez R, et al. Hypertension: prevalence, early diagnosis, control and trends in Mexican adults. Salud Publica Mex 2013;55(Suppl 2):S144-50.

8. World Health Organization. A global brief on hypertension. Silent killer, global public health crisis, 2013. http://www.who.int/ cardiovascular_diseases/publications/global_brief_hypertension/en/.
9. Lin CL, Liu TC, Lin FH, et al. Association between sleep disorders and hypertension in Taiwan: a nationwide population-based retrospective cohort study. J Hum Hypertens 2017;31:220-4.

10. Havranek EP, Mujahid MS, Barr DA, et al. . Social Determinants of Risk and outcomes for Cardiovascular Disease: a Scientific Statement from the American Heart Association. Circulation 2015;132:873-98.

11. Padmanabhan S, Caulfield M, Dominiczak AF. Genetic and molecular aspects of hypertension. Circ Res 2015;116:937-59.

12. Haffner SM. Hypertension in the San Antonio Heart Study and the Mexico City Diabetes Study: clinical and metabolic correlates. Public Health Rep 1996;111(Suppl 2):11-14.

13. INEGI. Censo de población y vivienda 2010. 2010. http://www.inegi. org.mx/est/contenidos/proyectos/ccpv/cpv2010.

14. Chow SC, Shao J, Wang H. Sample size calculations in clinical research. 2nd ed: Chapman \& Hall/CRC, 2008.

15. Pereira M, Lunet N, Paulo C, et al. Incidence of hypertension in a prospective cohort study of adults from Porto, Portugal. BMC Cardiovasc Disord 2012;12:114.

16. Takase H, Sugiura T, Kimura G, et al. Dietary sodium consumption predicts future blood pressure and incident hypertension in the japanese Normotensive General Population. J Am Heart Assoc 2015; 4:e001959.

17. Ford ES, Cooper RS. Risk factors for hypertension in a national cohort study. Hypertension 1991;18:598-606.

18. Chobanian AV, Bakris GL, Black HR, et al. Seventh report of the joint national committee on prevention, detection, evaluation, and treatment of high blood pressure. Hypertension 2003;42:1206-52.

19. Secretaría de Salud. Norma Oficial Mexicana NOM-015-SSA2-2010, para la prevención, tratamiento y control de la diabetes mellitus. 2010. http://www.spps.gob.mx/images/stories/SPPS/Docs/nom/ NOM-015-SSA2-2010.pdf.

20. The International Society for the Advancement of Kinanthropometry. International Standards for Anthropometric Assessment. Australia: ISAK, 2001.

21. SIDESO. Sistema de Información del Desarrollo Social - Índice del desarrollo social de las unidades territoriales del Distrito Federal. 2010 http://www.sideso.df.gob.mx/index.php?id=551.

22. Craig CL, Marshall AL, Sjöström M, et al. International physical activity questionnaire: 12 -country reliability and validity. Med Sci Sports Exerc 2003;35:1381-95.

23. Spielberger CD. Conceptual and methodological issues in anxiety research. Spielberger CD, ed. Anxiety: current trends in Theory and Research. . New York: Academic Press, 1972:2. 481-93.

24. Hays RD, Martin SA, Sesti AM, et al. Psychometric properties of the Medical Outcomes Study Sleep measure. Sleep Med 2005:6:41-4.

25. Spritzer K, Hays R. MOS sleep scale: a manual for use and scoring. Los Angeles : CA: RAND, 2003.

26. Hernández-Avila M, Romieu I, Parra S, et al. Validity and reproducibility of a food frequency questionnaire to assess dietary intake of women living in Mexico City. Salud Publica Mex 1998;40:133-40.

27. Hernández-Avila JE, González-Avilés L, Rosales-Mendoza E. Manua De Usuario. SNUT Sistema de evaluación de hábitos nutricionales y consumo de nutrimentos. Mexico City: Instituto Nacional de Salud Pública, 2003.

28. National Cholesterol Education Program, National Heart, Lung, and Blood Institute, National Institutes of Health. Detection, evaluation, and treatment of high blood cholesterol in adults (Adult treatment panel III). 2001.. http://www.nhlbi.nih.gov/files/docs/guidelines/ atp3xsum.pdf.

29. Centers for Disease Control and Prevention (CDC). State-specific secondhand smoke exposure and current cigarette smoking among adults - United States, 2008. MMWR Morb Mortal Wkly Rep 2009;58:1232-5.

30. Wielgosz A, Robinson C, Mao Y, et al. The impact of using different methods to assess completeness of 24-Hour urine collection on estimating Dietary sodium. J Clin Hypertens 2016;18:581-4.

31. Lahiri DK, Nurnberger Jl. A rapid non-enzymatic method for the preparation of HMW DNA from blood for RFLP studies. Nucleic Acids Res 1991;19:5444.

32. Martínez-Ríos MA, Alvarez-León E, Totomoch A, et al. Haplotypes of the angiotensin-converting enzyme (ACE) gene are associated with coronary artery disease but not with restenosis after coronary stenting. Exp Mol Pathol 2014;97:166-70.

33. O'Brien E, Atkins N, Stergiou G, et al. European Society of hypertension International Protocol revision 2010 for the validation of blood pressure measuring devices in adults. Blood Press Monit 2010;15:23-38.

34. Intersalt: an international study of electrolyte excretion and blood pressure. Results for 24601 hour urinary sodium and 
potassium excretion. Intersalt Cooperative Research Group. BMJ 1988;297:319-28.

35. Mente A, Dagenais G, Wielgosz A, et al. Assessment of Dietary sodium and potassium in Canadians using 24-Hour urinary collection. Can J Cardiol 2016;32:319-26.

36. Cappuccio FP, Stranges S, Kandala NB, et al. Gender-specific associations of short sleep duration with prevalent and incident hypertension: the Whitehall II Study. Hypertension 2007;50:693-700.

37. Fernandez-Mendoza J, Vgontzas AN, Liao D, et al. Insomnia with objective short sleep duration and incident hypertension: the Penn State Cohort. Hypertension 2012;60:929-35.

38. Gangwisch JE, Malaspina D, Posner K, et al. Insomnia and sleep duration as mediators of the relationship between depression and hypertension incidence. Am J Hypertens 2010;23:62-9.

39. Gangwisch JE, Heymsfield SB, Boden-Albala B, et al. Short sleep duration as a risk factor for hypertension: analyses of the first National Health and Nutrition Examination Survey. Hypertension 2006;47:833-9.

40. Lopez-Garcia E, Faubel R, Guallar-Castillon P, et al. Self-reported sleep duration and hypertension in older spanish adults. J Am Geriatr Soc 2009;57:663-8.

41. Wu X, Sun Y, Niu K, et al. Association of self-reported sleep duration and hypertension: results of a Chinese prospective cohort study. Clin Exp Hypertens 2016;38:514-9.

42. Fang J, Wheaton AG, Keenan NL, et al. Association of sleep duration and hypertension among US adults varies by age and sex. Am J Hypertens 2012;25:335-41.

43. Gottlieb DJ, Redline S, Nieto FJ, et al. Association of usual sleep duration with hypertension: the Sleep Heart Health Study. Sleep 2006;29:1009-14.

44. Priou P, Le Vaillant M, Meslier N, et al. Cumulative association of obstructive sleep apnea severity and short sleep duration with the risk for hypertension. PLoS One 2014;9:e115666.

45. Vozoris NT. Insomnia symptom frequency and hypertension risk: a population-based study. J Clin Psychiatry 2014;75:616-23.

46. World Health Organization. WHO | Social determinants of health. 2017. http://www.who.int/social_determinants/en/.

47. Feldman JM, Waterman PD, Coull BA, et al. Spatial social polarisation: using the index of concentration at the extremes jointly for income and race/ethnicity to analyse risk of hypertension. $J$ Epidemiol Community Health 2015;69:1199-207.

48. Leigh JP, Du J. Hypertension and occupation among seniors. J Occup Environ Med 2009;51:661-71.

49. Liu X, Gu W, Li Z, et al. Hypertension prevalence, awareness, treatment, control, and associated factors in Southwest China: an update. J Hypertens 2017;35:637-44.
50. Rodriguez F, Ferdinand KC. Hypertension in minority populations: new guidelines and emerging concepts. Adv Chronic Kidney Dis 2015;22:145-53.

51. Baek TH, Lee HY, Lim NK, et al. Gender differences in the association between socioeconomic status and hypertension incidence: the Korean Genome and Epidemiology Study (KoGES). BMC Public Health 2015;15:852.

52. Vijayaraghavan $M$, Kushel MB, Vittinghoff $E$, et al. Housing instability and incident hypertension in the CARDIA cohort. J Urban Health 2013;90:427-41.

53. Wang EA, Pletcher M, Lin F, et al. Incarceration, incident hypertension, and access to health care: findings from the coronary artery risk development in young adults (CARDIA) study. Arch Intern Med 2009;169:687.

54. Eaton CB, Feldman HA, Assaf AR, et al. Prevalence of hypertension, dyslipidemia, and dyslipidemic hypertension. J Fam Pract 1994;38:17-23.

55. Dalal JJ, Padmanabhan TN, Jain P, et al. LIPITENSION: Interplay between dyslipidemia and hypertension. Indian $J$ Endocrinol Metab 2012;16:240.

56. Onat A, Hergenç G, Sari I, et al. Dyslipidemic hypertension: distinctive features and cardiovascular risk in a prospective population-based study. Am J Hypertens 2005;18:409-16.

57. Halperin RO, Sesso HD, Ma J, et al. Dyslipidemia and the risk of incident hypertension in men. Hypertension 2006;47:45-50.

58. Freitas MP, Loyola Filho Al, Lima-Costa MF. Dyslipidemia and the risk of incident hypertension in a population of community-dwelling Brazilian elderly: the Bambuí Cohort Study of Aging. Cad Saude Publica 2011;27 Suppl 3:s351-s359.

59. Aguilar-Salinas CA, Gómez-Pérez FJ, Rull J, et al. Prevalence of dyslipidemias in the Mexican National Health and Nutrition Survey 2006. Salud Publica Mex 2010;52(Suppl 1):S44-S53.

60. Whelton PK, Appel LJ, Sacco RL, et al. Sodium, blood pressure, and cardiovascular disease: further evidence supporting the American Heart Association sodium reduction recommendations. Circulation 2012;126:2880-9.

61. World Medical Association. Declaración De Helsinki de la AMM - Principios éticos para las investigaciones médicas en seres humanos. 2013 http://www.wma.net/es/30publications/10policies/ b3/.

62. Secretaría de Salud. Reglamento de la Ley General de Salud en Materia de Investigación para la Salud. 2014 http://www.dof.gob.mx/ nota_detalle.php?codigo=5339162\&fecha=02/04/2014.

63. Bookman EB, Langehorne AA, Eckfeldt JH, et al. Reporting genetic results in research studies: summary and recommendations of an NHLBI working group. Am J Med Genet A 2006;140:1033-40. 
Correction: Protocol for a prospective longitudinal study of risk factors for hypertension incidence in a Mexico City population: the Tlalpan 2020 cohort

Colín-Ramírez E, Rivera-Mancía S, Infante-Vázquez O, et al. Protocol for a prospective longitudinal study of risk factors for hypertension incidence in a Mexico City population: the Tlalpan 2020 cohort. BMJOpen 2017;7:e016773. doi:10.1136/bmjopen-2017-016773

Reference 2 should read:

Danaei G, Finucane MM, Lin JK, et al. National, regional, and global trends in systolic blood pressure since 1980: systematic analysis of health examination surveys and epidemiological studies with 786 country-years and $5 \cdot 4$ million participants. Lancet 2011;377:568-77. doi:10.1016/S0140-6736(10)62036-3

Open Access This is an Open Access article distributed in accordance with the Creative Commons Attribution Non Commercial (CC BY-NC 4.0) license, which permits others to distribute, remix, adapt, build upon this work non-commercially, and license their derivative works on different terms, provided the original work is properly cited and the use is non-commercial. See: http://creativecommons.org/licenses/by-nc/4.0/

(C) Article author(s) (or their employer(s) unless otherwise stated in the text of the article) 2017. All rights reserved. No commercial use is permitted unless otherwise expressly granted.

BMJ Open 2017;7:e016773corr1. doi:10.1136/bmjopen-2017-016773corr1

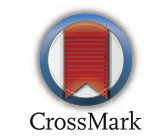

